\title{
Molecular Classification of Diffuse Gliomas
}

\author{
Navya Kalidindi†, Rosemarylin Or†, Sam Babak, Warren Mason
}

\begin{abstract}
Technological advances in the field of molecular genetics have improved the ability to classify brain tumors into subgroups with distinct clinical features and important therapeutic implications. The World Health Organization's newest update on classification of gliomas (2016) incorporated isocitrate dehydrogenase 1 and 2 mutations, ATRX loss, 1p/19q codeletion status, and TP53 mutations to allow for improved classification of glioblastomas, low-grade and anaplastic gliomas. This paper reviews current advances in the understanding of diffuse glioma classification and the impact of molecular markers and DNA methylation studies on survival of patients with these tumors. We also discuss whether the classification and grading of diffuse gliomas should be based on histological findings, molecular markers, or DNA methylation subgroups in future iterations of the classification system.
\end{abstract}

RÉSUMÉ : La classification moléculaire des gliomes diffus. Les avancées technologiques dans le domaine de la génétique moléculaire ont permis d'améliorer notre capacité à répertorier les tumeurs cérébrales dans des sous-groupes de tumeurs possédant chacun des caractéristiques cliniques différentes et sous-tendant d'importantes implications thérapeutiques. En vue de faciliter une classification améliorée des glioblastomes et des gliomes anaplasiques de bas grade, la plus récente mise à jour de l'OMS en matière de classification des gliomes (2016) a inclus les mutations des gènes IDH1 et IDH2, une perte d'expression du gène ATRX, une suppression simultanée du bras court du chromosome 1 et du bras long du chromosome 19 ainsi que ses effets, de même que les mutations du gène TP53. Cette étude entend donc passer en revue les avancées actuelles de la connaissance en ce qui concerne la classification des gliomes diffus. Elle entend aussi se pencher sur l'impact des marqueurs moléculaires et des études de méthylation de l'ADN quant à la survie des patients atteints de ces tumeurs. Nous examinerons également dans quelle mesure la classification et la notation des gliomes diffus dans les systèmes de classification à venir devraient être fondées sur des résultats histologiques, des marqueurs moléculaires ou des sous-groupes de méthylation de l'ADN.

Keywords: Molecular genetics, Glioma, Brain tumors, Molecular biology, Clinical neurogenetics, Neuro-oncology-medical, Glioblastoma, Oligodendroglioma, Astrocytoma, Molecular classification

doi:10.1017/cjn.2020.10

Can J Neurol Sci. 2020; 47: 464-473

\section{INTRODUCTION}

Glioblastoma (GBM) is the most malignant primary brain tumor and accounts for nearly $15 \%$ of all central nervous system (CNS) tumors, while astrocytic and oligodendroglial tumors (diffuse and anaplastic) account for approximately $7 \%$ of all primary CNS tumors. ${ }^{1}$ In diffuse gliomas, survival outcomes, response to treatment, and clinical characteristics of patients vary by the tumor grade and molecular alterations. ${ }^{2}$ While the 2007 World Health Organization Classification of Tumours of the Central Nervous System largely separated glioma types based on histological features ${ }^{3}$, the newest revision (from 2016) integrated molecular markers such as isocitrate dehydrogenase (IDH) 1 and 2 mutations, TP53 mutations, ATRX loss, and 1p/19q codeletion in classifying diffuse gliomas. ${ }^{4}$

Critical gene alterations leading to glioblastomas have been attributed to three pathways with varying degrees of frequencies. These include the RB1 pathway (68\%), the TP53 (64\%), and P13K/PTEN pathway (50\%). ${ }^{5}$ Glioblastomas can develop de novo, without lower grade precursors (primary GBM) or they can develop through the malignant transformation of lower grade tumors (secondary GBM). Grade II or III astrocytomas can transform into secondary GBM and diffuse oligodendrogliomas can transform to anaplastic oligodendrogliomas (AOs) ${ }^{6}$ It is not possible to distinguish secondary from primary glioblastomas by histology alone. ${ }^{7}$ Approximately $90 \%$ of glioblastomas are primary and $10 \%$ are secondary. ${ }^{8}$ There are distinct molecular identities for primary and secondary GBMs, astrocytomas, and oligodendrogliomas based on various coexisting mutations.

Over the last decade, the advent of DNA methylation studies and increasing knowledge of specific genetic alterations in glioma genomes has created the potential for classifying diffuse gliomas based on molecular features rather than histopathology alone. This paper reviews survival outcomes and clinical characteristics of patients based on IDH1/2 and other mutations typically seen in diffuse gliomas and based on DNA methylation studies. The classification system suggested by recent DNA methylation studies and glioma molecular markers will be discussed along with those of the current WHO 2016 tumor classification system.

From the Department of Medical Oncology and Hematology, University Health Network, Toronto, ON, Canada

Received May 2, 2019. Final Revisions Submitted December 29, 2019. Date of AcCePtance January 4, 2020.

Correspondence to: Warren Mason, Department of Medical Oncology and Hematology, University Health Network, Toronto, ON, Canada. Email: warren.mason@uhn.ca

$\dagger$ These authors contributed equally to this paper. 


\section{Removal of Previous Classification of Oligoastrocytoma}

Historically, a high degree of interobserver variability was noted in the diagnosis of gliomas, ${ }^{9}$ likely as a byproduct of primarily histological diagnosis rather than molecular grouping. Astrocytomas are known to harbor concurrent TP53 mutations (50-90\%) and ATRX mutations (loss of function mutations), while oligodendrogliomas have $1 \mathrm{p} / 19 \mathrm{q}$ codeletion (50-70\%), normal ATRX, and a much lower number of TP53 mutations $(5-10 \%) .{ }^{10,11}$ Prior to the most recent WHO brain tumour classifications system, oligoastrocytomas were defined as mixed Grade II gliomas. ${ }^{3}$ Maintz et al. showed that oligoastrocytomas without $1 \mathrm{p} / 19 \mathrm{q}$ codeletion often had TP53 mutations. ${ }^{12}$

The Cancer Genome Atlas Research Network used integrative genomic analysis to find three distinct genetic subtypes of lowgrade gliomas which were nonoverlapping and of prognostic significance. The first subtype included tumors with IDH mutations and $1 \mathrm{p} / 19 \mathrm{q}$ codeletions, and was found to have the best clinical outcome. The second group was IDH-mutated tumors, but with absent $1 \mathrm{p} / 19 \mathrm{q}$ codeletions. The third group was IDH wildtypes and exhibited poor clinical outcomes, similar to that of primary glioblastoma. ${ }^{13}$

The 2016 WHO Classification of Tumours of the Central Nervous System recommends that a diagnosis of oligoastrocytoma, not otherwise specified (NOS) and anaplastic oligoastrocytoma, NOS, be used only in exceptional situations involving gliomas exhibiting both astrocytic and oligodendroglial components that cannot be subjected to molecular testing or those with inconclusive molecular findings. ${ }^{14}$

\section{IDH Mutations}

\section{Role in Gliomagenesis}

Mutations in IDH enzymes have been implicated in some cancers such as acute myeloid leukemia, cholangiocarcinoma, myelodysplastic syndromes, and gliomas to name a few. ${ }^{15,16} \mathrm{Of}$ the three isozymes (IDH1, IDH2, and IDH3) which exist, IDH1 is in the cytosol and peroxisome while IDH2 and IDH3 are in the mitochondria. ${ }^{16,17}$ IDH mutations are typically heterozygous and involve arginine sites involved with the binding of isocitrate (namely the R132 in IDH1 and R172 and R140 codons in IDH2). ${ }^{16,18}$ They result in the conversion of alpha ketoglutarate (KG) to D-2-hydroxyglutarate (2-HG), which accumulates to generate epigenetic dysregulation through the inhibition of alpha KG-dependent histone and DNA methylases, thereby blocking cellular differentiation and promoting tumorigenesis. $5,16,18,19$

IDH mutations can occur in any grade of glioma (most frequently in Grade II/III astrocytomas and oligodendrogliomas as well as secondary glioblastomas) but are not seen in pilocytic astrocytomas. ${ }^{4}$ Most tumors with $1 \mathrm{p} / 19 \mathrm{q}$ codeletions have also been shown to have IDH mutations. ${ }^{20}$ IDH1 mutations occur very early in gliomagenesis, even prior to TP53 mutations and 1p/19q codeletions. ${ }^{21-23}$ Tumors without IDH1 mutation might instead have IDH2 R172 mutation. ${ }^{22}$ IDH1 and IDH2 mutations are in 80$88 \%$ of oligodendrogliomas. ${ }^{24}$ More than $70 \%$ of astrocytomas and oligodendrogliomas (WHO Grade II and Grade III) harbor the IDH1 R132 mutation. ${ }^{7}$ If the R132H mutation-specific antibody (most common) testing is negative, the patient may have a noncanonical mutation, which can be determined with DNA sequencing. ${ }^{25}$ IDH mutations are linked with the proneural phenotype and secondary glioblastomas, as well as increased DNA methylation (called G-CIMP for glioma CpG island methylation phenotype). ${ }^{6}$

In contrast to adult gliomas, pediatric gliomas do not typically harbor IDH mutations. ${ }^{26}$ In a study of 43 patients with high-grade gliomas, Pollack et al. found that $35 \%$ of children over the age of 14 years had an IDH1 mutation but those who were younger than this group had no IDH mutations. ${ }^{27}$ This suggests that pediatric gliomas may be unique molecular entities that differ markedly from adult gliomas.

\section{IDH1 Versus IDH2 Mutations}

In gliomas, IDH1 mutations are generally more common than IDH2 mutations and are known to be mutually exclusive. ${ }^{23}$ In their genomic analysis of GBM patients, Parsons et al. found that $12 \%$ of primary GBM patients had an IDH1 mutation and Wang et al. found that $14.1 \%$ of primary GBM patients had either IDH1/2 mutations. ${ }^{5,23}$ Hartmann et al. studied 1010 cases of diffuse gliomas and found that $73 \%$ of diffuse astrocytomas had an IDH1 mutations (compared to $0.9 \%$ with IDH2 mutations) and $64 \%$ of anaplastic astrocytomas (AAs) had IDH1 mutations (compared to $0.8 \%$ with IDH2 mutations). In the AO group, $69.5 \%$ had IDH1 mutations while $5.2 \%$ had IDH2 mutations. There were IDH1 mutations in $82 \%$ and IDH2 mutations in $4.7 \%$ of diffuse oligodendrogliomas. Of note, this study was performed prior to the newest WHO brain tumor classification (2016) and thus a separate group of "oligoastrocytomas" was also discussed in the original article. The IDH2 mutations were highly associated with $1 \mathrm{p} / 19 \mathrm{q}$ codeletions, as per a recent study by Wang et al., who found that $44.4 \%$ of IDH2-mutated gliomas had a $1 \mathrm{p} / 19 \mathrm{q}$ codeletion. In addition, IDH2 mutations in gliomas are mutually exclusive to other mutations typically seen in gliomas (PTEN, TP53, ATRX). ${ }^{23}$

\section{Clinical Significance of IDH Mutations in Gliomas}

In a study by Yan et al., patients with IDH-wildtype GBMs were reported to be older than their IDH-mutated counterparts (59 versus 32 years) and are thought to have worse prognosis; similarly, IDH-wildtype AA patients were older than IDHmutated AA patient counterparts (56 versus 34 years). ${ }^{7}$ With regard to treatment response, $\mathrm{Li}$ et al. suggested that gliomas with IDH mutations are more sensitive to radiation therapy while other studies suggested that IDH-mutated gliomas may have a higher response to alkylating agents. ${ }^{28-30}$

Grade II-IV gliomas with IDH mutations have better survival than those without, suggesting the prognostic significance of this mutation, independent of other factors such as tumor grade and $\mathrm{O}^{6}$-methylguanine-DNA-methyltransferase (MGMT) methylation. $^{31}$ This trend for improved survival with IDHmutated gliomas when compared to their non-IDH-mutated counterparts was also noted by several other studies in gliomas. ${ }^{7,24,32-34}$ Weller et al. demonstrated a progression-free survival benefit in GBMs with IDH mutations compared to those with IDH-wildtype status (16.2 versus 6.5 months) and a trend toward benefit for overall survival (30.2 versus 11.2 months). ${ }^{34}$ Though there may be a progression-free survival benefit for IDH2 mutations when compared to IDH1 mutations, overall survival is not thought to be different between the two groups of mutations. ${ }^{23}$ Several other studies have shown that IDH1 and IDH2 mutations predict longer survival in secondary glioblastomas and 
Table 1: Common mutations seen in IDH-wildtype low-grade gliomas versus IDH-wildtype glioblastomas

\begin{tabular}{l|c}
\hline IDH-wildtype low-grade gliomas & IDH-wildtype GBM \\
\hline EGFR $(14 \%)$ & TERT $(72-90 \%)$ \\
\hline TERT $(27 \%)$ & PTEN $(25-35 \%)$ \\
\hline BRAF $(7 \%)$ & EGFR $(35-45 \%)$ \\
\hline H3F3A $(9.5 \%)$ & CDKN2A/2B $(60 \%)$ \\
\hline & TP53 $(28-35 \%)$ \\
\hline
\end{tabular}

Grade II and Grade III gliomas. ${ }^{29,30}$ Parsons et al. found that GBM patients with IDH1 mutations had an improved overall survival of 3.8 years (45.6 months) compared to the 1.1 years (13.2 months) noted in those without IDH1 mutations. The survival benefit was noted independent of an additional TP53 mutation. ${ }^{5}$ IDH mutations were associated with improved median survival for both GBM (median overall survival [OS] of 31 versus 15 months in those who were IDH wildtype) and AA (median OS of 65 versus 20 months without mutations). ${ }^{7}$

In summary, IDH mutations occur early in gliomagenesis and most commonly occur in low-grade and AAs and oligodendrogliomas as well as in secondary glioblastomas. Though multiple studies have addressed the possibility of classifying gliomas by their IDH status (rather than by histology and IDH status, as per current WHO guidelines), there is no clear evidence that IDH status alone determines tumor grade as prognosis is also dependent on other concurrent mutations associated with gliomas. These mutations will be discussed in the next section.

\section{Other Common Genetic Alterations in Diffuse Gliomas}

IDH-wildtype GBMs have higher rates of PTEN, EGFR, CDKN2A, or CDKN2B mutations while IDH-wildtype low-grade gliomas can have EGFR amplification, TERT (telomerase reverse transcriptase) mutations, and BRAF mutations (Table 1). ${ }^{35}$ Of note, while H3F3A mutations have been shown to confer worse prognosis, Aibaidula et al..$^{35}$ found that they occur in $9.5 \%$ of lowgrade gliomas but are not relatively as common in IDH-wildtype glioblastomas. The most common genetic abnormalities in IDHwildtype glioblastomas are TERT mutations (72-90\%), EFGR amplifications (35-45\%), homozygous deletion of CDKN2A/ CDKN2B (60\%), loss of chromosomes 10p or of 10q (50\% and $70 \%$, respectively), TP53 mutation (28-35\%), and PTEN mutations (25-35\%). ${ }^{4}$ In contrast, IDH-mutant GBMs are more often associated with a hypermethylator phenotype, originate from a proneural transcriptional profile, and have typical genetic alterations such as TP53 (81\%), ATRX mutation (71\%), loss of chromosome arm 19q (50\%), loss of chromosome arm 10q $(>60 \%)$, and TERT mutations $(30 \%){ }^{4}$ In addition, IDH-mutant GBMs often lack EGFR amplification, which is more consistent with secondary glioblastomas. ${ }^{34,36}$ EGFR amplification and PTEN mutations are extremely rare in the IDH-mutated GBM population and TERT mutations are less frequently seen $(26 \%) .{ }^{4}$ In lower grade gliomas (IDH wildtype), Aibaidula et al. found that $26.8 \%$ of cases had TERT, $13.8 \%$ had EFGR amplification, $6.9 \%$ had BRAF mutations, and $9.5 \%$ had H3F3A mutations. ${ }^{35}$ EGFR alterations and PTEN deletions are more commonly seen in primary GBMs than secondary GBMs. ${ }^{8}$
However, there are rare histological variants of IDH-wildtype GBMs whose genetic profiles differ from the typical IDHwildtype GBMs. Giant cell glioblastomas are characterized by bizarre-looking multinucleated giant cells. While this variant frequently possesses PTEN and TP53 mutations, they typically lack EGFR amplification/overexpression and homozygous CDKN2A deletion. ${ }^{37}$ Their clinical prognosis is fairly better compared to the more typical IDH-wildtype GBM. ${ }^{37}$ Another variant of IDHwildtype GBM is made up of closely packed epithelioid cells, aptly named epithelioid glioblastoma. These are aggressive tumors, even compared to an ordinary GBM and about $50 \%$ of its population contain BRAF V600E mutation. ${ }^{38}$ The third IDH-wildtype variants are gliosarcomas, which are characterized by alternating areas of glial and mesenchymal differentiation. They contain the typical GBM PTEN mutations, CDKN2A deletions, and TP53 mutations, but only have infrequent EGFR amplifications. Gliosarcomas have the same outcome as a classic GBM, but systemic metastasis and skull invasions had been reported for this variant. ${ }^{39}$

\section{1p/19q Codeletion}

In the 2016 WHO classification update, oligodendrogliomas are molecularly defined by IDH mutations with concurrent codeletion of chromosomes 1p and 19q. They often have TERT mutations but ATRX loss is not present. Testing of $1 \mathrm{p} / 19 \mathrm{q}$ codeletion is often completed through FISH (fluorescence in situ hybridization), among other methods of testing currently available. ${ }^{40}$ However, detection of $1 \mathrm{p} / 19 \mathrm{q}$ codeletion by FISH is not always accurate and can lead to false positives (e.g., when FISH is positive in the event of interstitial deletions involving the areas with probe hybridization or with monosomy, without complete deletion of the $1 \mathrm{p}$ and $19 \mathrm{q}$ chromosomes). ${ }^{41}$ This potential for false classification using FISH testing for this codeletion could be corrected using more detailed testing such as chromosomal microarray. ${ }^{41}$ In addition, $1 \mathrm{p} / 19 \mathrm{q}$ codeletion can also be found in glioblastomas and astrocytic tumors, presenting a diagnostic challenge. DNA methylation studies could assist in properly classifying tumors in these cases (discussed in a separate section). The presence of this chromosomal codeletion of $1 \mathrm{p}$ and $19 \mathrm{q}$ has been shown to confer a significant survival advantage in patients who were treated with radiotherapy and Procarbazine, Lomustine, Vincristine (PCV) chemotherapy. ${ }^{42,43}$

\section{ATRX Mutations}

If a tumor is negative for a $1 \mathrm{p} / 19 \mathrm{q}$ codeletion in the setting of IDH-wildtype status, it is likely an IDH-wildtype AA or glioblastoma ${ }^{44}$ In contrast, astrocytomas also have IDH mutations but with loss of ATRX - they do not typically have TERT promotor mutations or $1 \mathrm{p} / 19 \mathrm{q}$ codeletions. ${ }^{4}$ ATRX mutations lead to lack of expression of ATRX and are typically associated with IDH1/2 mutations and TP53 mutations in Grade II and III astrocytomas and IDH-mutated GBM. ${ }^{4}$ ATRX retention with $1 \mathrm{p} /$ $19 \mathrm{q}$ codeletion confers a better prognosis in anaplastic gliomas. ${ }^{4}$ Wiestler et al. reported better progression-free survival in IDHmutated astrocytic tumors with ATRX loss (55.6 months) compared to those without ATRX loss (31.8 months). ${ }^{45}$

\section{TP53 Mutations}

TP53 is a tumor suppressor gene and mutations lead to overexpression of p53 as a gain-of-function mechanism. ${ }^{4} \mathrm{~A}$ high 
frequency of TP53 mutations in GBM patients with IDH1 mutations has been reported. ${ }^{5}$ Cohen et al. found that $80 \%$ of IDH-mutated AAs and IDH-mutated glioblastomas have TP53 mutations. ${ }^{6}$ These mutations are also present in diffuse astrocytic tumors but are not prognostic of clinical outcomes. ${ }^{46}$ In contrast, only $9 \%$ of AOs have been reported to have TP53 mutations. ${ }^{7}$ An update in 2018 by the Consortium to Inform Molecular and Practical Approaches to CNS Tumor Taxonomy (cIMPACTNOW) recommended that an IDH-mutated Grade II or III astrocytoma can be diagnosed in the setting of an astrocyticlooking tumor that expressed IDH mutation, loss of ATRX, and/or a diffuse p53 positivity, even when $1 \mathrm{p} / 19 \mathrm{q}$ testing is absent. $^{47}$ This update allows for further improvements in the classification of astrocytomas since the 2016 WHO criteria.

\section{TERT (Telomerase Reverse Transcriptase)}

TERT promoter mutations increase promoter activity and are seen in glioblastomas (up to $80 \%$ ) and oligodendrogliomas. These mutations are rarely found in astrocytomas or secondary (IDH-mutated) glioblastomas but are often found in IDHwildtype glioblastomas. ${ }^{4}$ TERT promoter mutations are inversely correlated with frequency of IDH and TP53 mutations in glioblastomas. ${ }^{48}$ TERT promotor mutations and ATRX mutations were found to be mutually exclusive in most cases. ${ }^{49}$ TERT mutations are often associated with aggressive clinical course in a diffuse astrocytic gliomas. The 2018 c-IMPACT-NOW update suggests that it is best to test for IDH mutation and $1 p / 19 q$ codeletion status to rule out the diagnosis of oligodendroglioma as most oligodendrogliomas are known to exhibit TERT promoter mutation as well. ${ }^{13}$ Age at time of diagnosis, molecular markers, and grade of tumor are independent associations with overall survival. ${ }^{49}$ Patients with GBM who have TERT and IDH mutations do not have better survival than those with TERT mutations only, suggesting that IDH mutations do not dictate prognosis alone and that TERT mutations hold independent prognostic value. ${ }^{49,35,50}$

\section{Exploring the Role of Tumor Stratification Based on IDH Mutation Status}

Several studies have suggested that histologically Grade II and Grade III tumors may have a dismal prognosis if associated with IDH-wildtype status. ${ }^{7,20,49}$ Metellus et al. studied 47 low-grade gliomas (18 oligodendrogliomas, 7 astrocytomas, and 22 oligoastrocytomas - as per the WHO 2007 classification system) and found that absence of IDH1/2 mutations, older age, lower extent of surgery, and frontotemporoinsular tumors with a highly infiltrative pattern were poor prognostic factors. These authors found that low-grade gliomas with IDH1/2 mutations had a median PFS of 4.7 years (56.4 months), while IDH1/2-wildtype tumors had median progression free survival (PFS) of 1.4 years (16.8 months). Reuss et al. ${ }^{51}$ analyzed $160 \mathrm{IDH}$-wildtype Grade II (40/160) and Grade III (120/160) astrocytomas (by WHO 2007 classification system) and found that $78 \%$ of these tumors could be reclassified as glioblastomas by molecular analysis, while $17 \%$ had a molecular profile consistent with GBM-H3F3A or GBMH3-K27 subtypes. Molecular subtypes in this study were determined by G-CIMP phenotype testing and determination of copy number alterations on $450 \mathrm{k}$ methylation analysis as well as sequencing for IDH1/2, H3F3A, and TERT promoter mutations. Interestingly, of the $78 \%$ of tumors reclassified as GBMs (as per hallmark genetic alterations, DNA methylation profiles, and copy number profiles), most also had at least a TERT promoter mutation, 10q loss and 7q gain, and EGFR amplification (findings often associated with IDH-wildtype GBMs). True to the molecular diagnosis, the median survival for the molecular GBM subgroup was approximately 19.4 months. In summary, the authors found that most IDH-wildtype astrocytomas determined by histology could be reclassified as GBMs by molecular basis and have a similar median overall survival to conventional GBMs.

Other studies contradict this and suggest that the IDH status alone does not supersede the histological grading criteria as the presence of several associated mutations also contributes to the overall survival. $^{35,52}$ Chan et al. ${ }^{53}$ studied 214 lower grade gliomas and found that median overall survival was most favorable for those with IDH1/2 mutation along with either $1 p / 19 q$ codeletion or a TERT mutations, while it was least favorable for those with IDH-wildtype status and a TERT mutation or EGFR amplification. However, they found that the histological grade in these cases was still more representative of survival outcomes than the mutations in tumors within each grade. Aibaidula et al. analyzed 718 Grade II and Grade III gliomas (per WHO 2007 classification) and found that a molecularly higher grade of tumors (IDH-wildtype, EGFR, H3F3A, or TERT mutations) had median overall survival of 1.23 years (14.76 months), whereas the "molecularly lower grade" tumors (IDH mutated with no EGFR, TERT, or H3F3A mutations) had a significantly higher overall survival of 7.63 years ( 91.56 months). These tumors had higher survival than the approximately 30 -month overall survival reported in IDH-mutant glioblastomas who undergo standard of care chemotherapy. ${ }^{34}$ A more recent meta-analysis by Vuong et al. also demonstrated a propensity for IDH-wildtype low-grade gliomas to have a poor prognosis when associated with TERT promoter mutations, EGFR amplifications, and H3F3A mutations, whereas ATRX mutation, $7 \mathrm{q}$ gain/10q loss, and CDKN loss did not influence prognosis. ${ }^{54}$

Thus, there is not enough evidence that IDH status alone should dictate the classification of glioma; instead, IDH status must be taken in conjunction with other prognostic mutations commonly found in gliomas in addition to the clinical and radiological status of the patient. This is reflected in the cIMPACT-NOW consortium guidelines (2018) which designate IDH-wildtype Grade II or III tumors, with one of the following molecular characteristics: EGFR amplification, or combined whole chromosome 7 gain and whole chromosome 10 loss, or TERT promoter mutation, as "diffuse astrocytic glioma, IDH-wildtype, with molecular features of glioblastoma, WHO grade IV." 55 The clinical implications of this new designation mean that a combination of chemotherapy and radiotherapy is recommended for these types of tumors. In addition, inclusion criteria for clinical trials may be extended to include this group of patients. ${ }^{55}$

\section{BRAF fusions versus mutations}

BRAF is an intracellular serine/threonine kinase component of the mitogen-activated protein kinase pathway. It is involved in the activation of transcription factors that induces cell proliferation, survival, and tumorigenesis - it can also trigger 
differentiation and senescence. ${ }^{56,57}$ BRAF is the more potent activator of MEK compared to other RAF isoforms and has a greater oncogenic potential. The most frequent BRAF mutations found in gliomas are BRAF gene rearrangements and fusions. ${ }^{56}$

\section{BRAF fusions}

A tandem duplication and rearrangement on chromosome $7 q 34$ between BRAF and a gene, KIAA1549, produces a fusion gene. ${ }^{56}$ KIAA1549:BRAF fusion is detected in about $80 \%$ of pediatric pilocytic astrocytomas, but rarely in low-grade diffuse astrocytomas. $^{58,59}$ Because pilocytic astrocytomas frequently harbor BRAF fusions, it has been suggested that along with other markers, the presence of this fusion may help support the diagnosis of pilocytic astrocytoma. ${ }^{60}$ The presence of the BRAF fusion gene was shown to be associated with a better overall survival in pilocytic astrocytomas. ${ }^{58}$ Despite its uncertain diagnostic value, the detection of KIAA1549:BRAF fusion may be useful in identifying low-grade astrocytic glioma patients with a longer survival outcome. ${ }^{59}$

\section{BRAF V600E mutation}

BRAF V600E mutations account for $90 \%$ of mutations in the BRAF gene. ${ }^{57}$ The V600E mutation was found in two-thirds of pleomorphic xanthoastrocytomas (PXA), and in approximately $20 \%$ of gangliogliomas in a series of 1320 adult and pediatric tumor. ${ }^{61}$ Similar results were found by Horbinski, with BRAF V600E mutations being found in $73.8 \%$ of PXAs and $19.2 \%$ of gangliogliomas. ${ }^{56} \mathrm{~A}$ high percentage of epithelioid GBMs $(7 / 13,53.8 \%)$ also harbor V600E mutation. Six out of the seven BRAF-mutated epithelioid GBM cases were observed in adults. ${ }^{62}$ Despite its high frequency in epithelioid GBMs, only $7.7 \%$ of adult GBMs showed BRAF V600E immunopositivity. ${ }^{63}$ Despite the high incidence of BRAF V600E mutations in the tumor groups mentioned earlier, it is important to keep in mind that the absence of this mutation does not exclude a histological diagnosis of these tumors. ${ }^{62}$

\section{Histone mutations}

The 2016 WHO classification identified a new entity called diffuse midline glioma, H3 K27M mutant. ${ }^{4}$ Histone H3 subgroups include G34R/V mutations (seen in pediatric high-grade gliomas of the cerebral hemispheres) and the K27M mutations (seen in the midline pediatric high-grade gliomas). ${ }^{64} \mathrm{G} 34 \mathrm{R} / \mathrm{V}$ histone mutations tend to occur in the cerebral hemispheres (not midline) and are mutually exclusive to the more common H3.3 K27M mutation.

H3 K27M mutations can be found in pediatric and adult diffuse intrinsic pontine gliomas (DIPG), adult diffuse midline gliomas (located in the thalamus, spinal cord, pons, and brainstem), gangliogliomas, and pilocytic astrocytomas. ${ }^{65,66}$ DIPGs were shown to have a median overall survival of 11.1 months and thalamic diffuse midline gliomas have been shown to have 10.8 month overall median survival. ${ }^{67}$

Several variations in histone mutations have been reported. Castel et al. have recently also found two subgroups of H3 K27M-mutant tumors, H3.1 K27M (HIST1H3B gene) and H3.3 K27M (H3F3A gene), which had distinct DNA methylation profiles, though prognostic difference between these two subtypes was not discussed in this paper. ${ }^{67} \mathrm{~K} 27 \mathrm{M}$ mutations in H3F3A or HIST1H3B genes have been shown in other studies to confer a worse prognosis for patients with midline glioma ${ }^{68,69}$, irrespective of age, histological grading, or tumor morphology. ${ }^{63}$ The H3F3A-mutated genotype demonstrated lower responses to radiotherapy increased metastatic spread and had earlier relapses when compared to the HIST1H3B-mutated genotype. ${ }^{70}$ The presence of an H3 G34 mutation in a diffuse astrocytic glioma indicates a high-grade biology, regardless of histological grade. ${ }^{41}$ As further research is conducted on the significance of histone mutations, there may be a role for further classifying midline gliomas based on these molecular aberrations.

In summary, distinct concurrent mutations and molecular profiles are often associated with IDH-wildtype and mutant gliomas with varying grades as described above. Presence of $1 \mathrm{p} / 19 \mathrm{q}$ codeletion confers a significant survival advantage in patients treated with radiation and chemotherapy. Similarly, a survival advantage has been shown in astrocytic tumors with IDH mutations and ATRX loss compared to those without ATRX loss. Mutations in the tumor suppressor gene, TP53, are common in IDH-mutated astrocytomas and IDH-mutated glioblastomas but are not of any known prognostic significance. TERT promoter mutations are inversely correlated with the presence of IDH and TP53 mutations and are thus more commonly seen in IDH-wildtype glioblastomas. TERT promoter mutations are also mutually exclusive to ATRX mutations and are thus not often seen in astrocytomas. BRAF mutations and fusions can be found in many tumors. In pilocytic astrocytomas, BRAF fusions are seen in $80 \%$ of tumors and confer a survival advantage. The BRAFV600E mutation accounts for $90 \%$ of BRAF gene mutations and is found in two-thirds of PXA and about half of epitheliod glioblastomas. Several variations of histone mutations have been reported thus far. H3 K27M mutation, which confers a survival disadvantage, has been included in the 2016 WHO classification system and is associated with pediatric and adult DIPG, gangliogliomas, and pilocytic astrocytomas.

\section{MGMT Methylation}

The MGMT gene is located on chromosome 10q26 and has a CpG-rich island with $98 \mathrm{CpG}$ sites encompassing most of the promoter and the first exon. It normally catalyzes the transfer of a methyl group from the $\mathrm{O}^{6}$ position of a guanine nucleotide to a cysteine residue at the 145 th position. ${ }^{71}$ It is a DNA repair gene, which must be replenished by the cell after being used in DNA restoration. $^{72}$

With the use of alkylating chemotherapeutic agents act by the binding of an alkyl group to the $\mathrm{O}^{6}-$ position of guanine. This induces DNA mismatching, double-strand breakage, and ultimately cellular apoptosis. High levels of MGMT in cancer cells make them more resistant to alkylating chemotherapy, resulting in treatment failure. Methylation of the cytosine in $\mathrm{CpG}$ sites increases its affinity to proteins that prevent binding of transcription factors, effectively silencing MGMT expression. The epigenetic silencing of MGMT gene diminishes the tumor cell's DNA repair capacity, making it more susceptible to the cytotoxic effects of alkylating chemotherapeutic agents. ${ }^{71,72}$

About half of all glioblastomas have methylated promoter sequences to the MGMT gene. ${ }^{72}$ MGMT promoter methylation is associated with longer survival (21.7-month median survival 
versus 15.3 months for those without methylation) and better responses to chemotherapy with alkylating agents. ${ }^{72,73}$ Secondary GBMs and astrocytomas with MGMT methylation are more likely to have a TP53 mutation and IDH mutations compared to those without methylation. ${ }^{74}$

\section{Use of DNA Methylation in Classification of Gliomas}

Integrating molecular markers and histological diagnoses into the 2016 WHO CNS tumor classification has allowed for better subtyping of gliomas but does not account for intra-tumoral heterogeneity within the same subtypes. ${ }^{75,76}$ The advent of DNA methylation profiling of gliomas allows for further refinement in diagnoses and integrating testing.

\section{Principles of DNA Methylation Profiling in Gliomas}

DNA methylation profiling involves identifying molecular difference among tumor groups as well as normal tissues. A hallmark of carcinogenesis is the disruption of normal gene regulation that leads to a loss or gain of function. Cytosine methylation is a stable epigenetic modification that may play a role in gene silencing. ${ }^{77}$ Promoter $\mathrm{CpG}$ island (CGI) hypermethylation results in gene silencing, characteristic of gliomas, and is often seen with global DNA hypomethylation. ${ }^{40,78}$ DNA methylation, histone modification, and nucleosome remodelling all play an integral role in heritable gene silencing. Changes in these processes can result in pathologic gene silencing found in cancer. ${ }^{79}$

Toyota et al. ${ }^{80}$ initially proposed the concept of a distinct $\mathrm{CpG}$ island methylation phenotype (CIMP) which was found in a subtypes of colorectal cancer. ${ }^{43}$ CIMP has since been used in studies in an attempt to refine glioma classifications. These studies will be discussed in the succeeding paragraphs of this paper.

Aside from studies of glioblastomas, genome-wide methylation profiling was also done in oligodendroglial tumors, with the work of van den Bent et al. showing CGI hypermethylation phenotype confers a better overall survival compared to unmethylated subgroup. ${ }^{81}$

Noushmehr and colleagues had characterized a distinct subset of gliomas based on the presence of a G-CIMP using DNA methylation testing. Proneural subtype of GBM was seen to have a highly enriched G-CIMP cluster, while mesenchymal and classical subtypes' G-CIMP clusters were found to be moderately enriched. Patients with G-CIMP-positive proneural tumors were noted to have a significantly younger age at onset and better survival compared to G-CIMP-negative proneural GBMs and other non-proneural GBM subtypes. IDH-1 mutation was seen to be tightly associated with G-CIMP positivity across all morphological glioma grades. G-CIMP positivity was observed to confer an improved survival outcome in these types of tumors. Independent of the IDH1 mutation status, G-CIMP has been shown to confer a favorable prognosis. ${ }^{78,82}$ The subtyping was studied further by Ceccarelli et al. ${ }^{82}$ in 2016 when they concluded that IDH-mutant gliomas may be divided into three subgroups, namely: (1) the Codel group, which is composed of IDH-mutated, 1p/19q-codeleted low-grade gliomas; (2) the G-CIMP-low group, which included IDH-mutated non-1p/19q-codeleted gliomas that have low levels of DNA methylation; and (3) the G-CIMP-high group, including IDH-mutated $1 \mathrm{p} / 19 \mathrm{q}$ non-codeleted gliomas with high levels of DNA methylation. They found that the G-CIMP-low group had a worse survival when compared to the $1 \mathrm{p} / 19 \mathrm{q}$-codeleted group and the G-CIMP-high group (log-rank $p$-value $\left.3.9 \times 10^{-10}\right){ }^{82}$ This showed that G-CIMP positivity conferred better survival outcomes independent of IDH mutation status.

Further molecular subtyping by Kloosterhof et al. divided gliomas into three groups based on their $1 \mathrm{p} / 19 \mathrm{q}$ codeletion status: the "C-" subtype (CIMP- tumors, most GBMs and all samples of pilocytic astrocytoma), the "C+1p19q" (CIMP+, most AOs and a few GBMs), and "C+wt" (CIMP+, histologically diverse, mostly AA). ${ }^{83}$ Median survival for the $\mathrm{C}-, \mathrm{C}+\mathrm{wt}$, and $\mathrm{C}+1 \mathrm{p} 19 \mathrm{q}$ subtypes were 1.18, 2.62, and 5.00 years (14.16, 31.44, and 60 months), respectively. The sample size of pilocytic astrocytomas was too small to identify a separate subgroup. This study demonstrated that these three subtypes of gliomas had significant prognostic factors based on their characteristic methylation profiling.

\section{CIMP on recurrence}

The possibility of the G-CIMP-high group serving as a predecessor of the G-CIMP-low tumor type was investigated by Ceccarelli et al. by comparing the DNA methylation profiles of 10 IDH-mutated, non-1p/19 q-codeleted low-grade gliomas and GBMs with the TCGA cohort. ${ }^{82}$ A clear (4 out of 10 cases) or partial (remaining 6 of 10 cases) demethylation pattern was noted after disease recurrence supporting the idea of a possible progression from a G-CIMP-high to a G-CIMP-low phenotype upon disease recurrence. ${ }^{82}$ De Souza et al ${ }^{84}$ followed this up with their 2018 study where they observed that while most IDHmutant LGG G-CIMP-high tumor patients retain their epimethyl phenotype on recurrence, there is a small proportion (17\%) that underwent progression to a G-CIMP-low phenotype on relapse. A distinct subgroup with G-CIMP-intermediate profiles was characterized by a modest degree of DNA methylation changes that trends toward G-CIMP-low subtype suggesting that this group of tumors may be an early-stage transition from a G-CIMP-high tumor to a G-CIMP-low tumor. The group had also found a set of predictive biomarkers signatures which are composed of seven hypomethylated CpG sites in initially G-CIMP-high tumors that progressed to GBMs on recurrence. They were also able to note that $95 \%$ of tumors previously classified as IDH-mutant non-1p/19qcodeleted G-CIMP-low tumors belong to this "risk group" that was prone to progress to a G-CIMP-low IDH-wildtype GBM-like phenotype on recurrence. ${ }^{84} \mathrm{We}$ made a summary of the subgroups proposed by these studies in Figure 1. Of note, this figure shows grouping by DNA methylation studies as well as by the current (2016) WHO classification criteria in such a way that there is overlap with methylation and histological diagnoses.

\section{GBM Molecular Subtypes by DNA Profiling}

With the advent of genome-wide DNA profiling, an increasing number of molecular subtypes are emerging. Phillips et al. ${ }^{85}$ were the first to coin the terms proneural, proliferative and mesenchymal to divide high-grade astrocytomas (AAs and glioblastomas) into molecular subclasses. They were able to observe that there is a mutually exclusive molecular markers expression pattern in the 


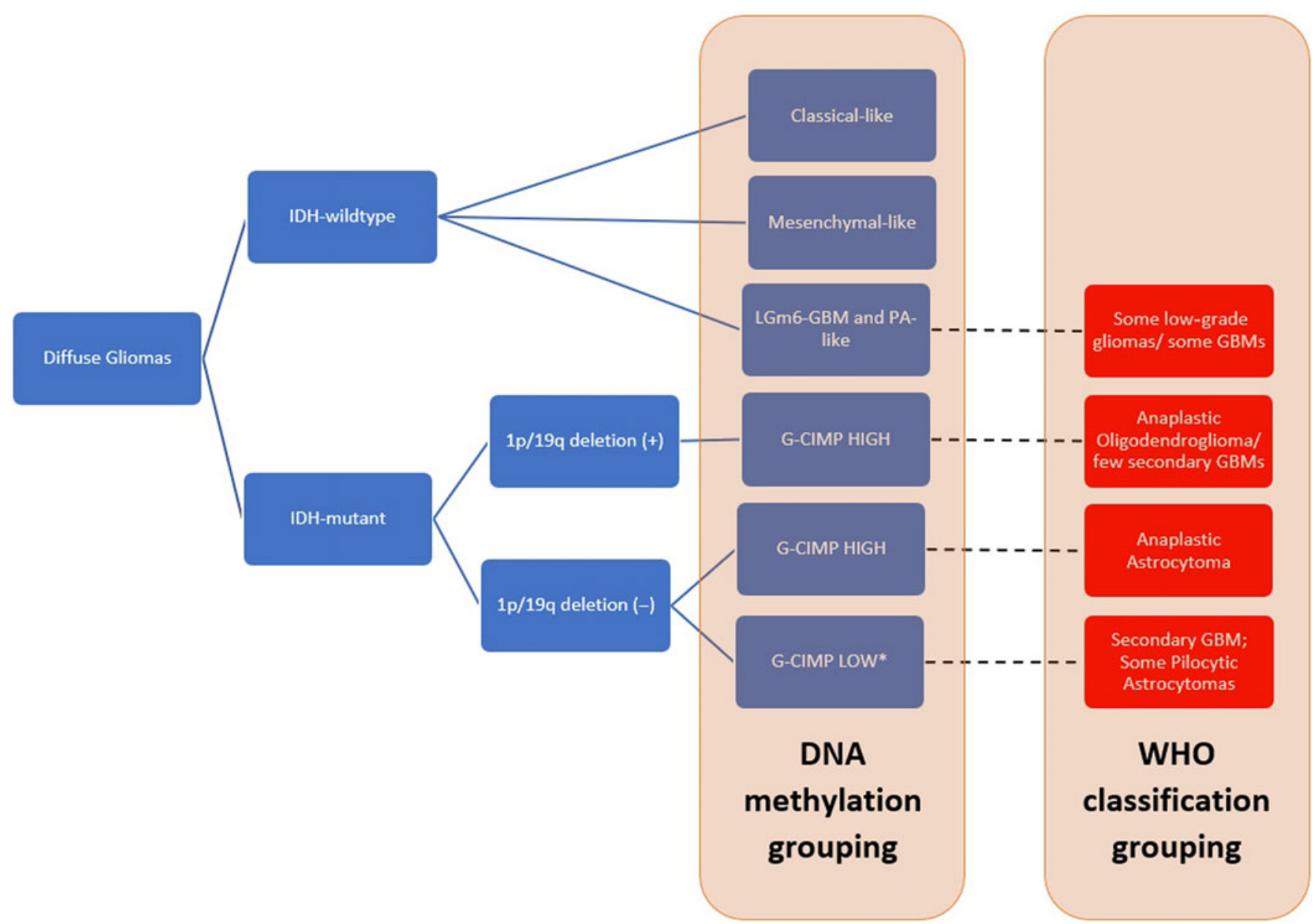

Figure 1: Summary of DNA methylation grouping based on the studies by de Souza et al., ${ }^{84}$ Ceccarelli et al.. ${ }^{82}$ and Kloosterhof et al. ${ }^{83}$ as well 2016 WHO classification grouping. The diagram also shows that there is some overlap of histological groups when classified via DNA methylation testing. Red boxes represent the tumor samples classified based on WHO classification criteria, showing overlaps in the tumor grouping. ${ }^{* M a j o r i t y ~(95 \%)}$ belong to a "risk group" that transforms into an IDH-wildtype, G-CIMP-low GBM-like tumor on relapse. ${ }^{84}$

proneural and mesenchymal subgroups. The proliferative signature is less exclusive compared to the other two subgroups, but the gain of the PIK3R3 locus on chromosome 1 appears to be a unique alteration in this type of tumor. Cases segregated into the proneural subclass were found to be younger compared to mesenchymal or proliferative subclasses. Median survival was noted to be longer in the proneural subclass (174.5 weeks) compared to either proliferative $(60.5$ weeks $)$ or mesenchymal subtypes (65.0 weeks) (Table 2).

While a mixture in Grade III and IV gliomas was found in the proneural subclass, Grade IV gliomas predominated the mesenchymal and proliferative groups. The presence of some cases of Grade IV tumors in the proneural subclass may serve as evidence that molecular classification might prove to be superior to histological grading in prognostication. ${ }^{85}$

The GBMs sorted into the poorest prognostic molecular subgroups have a significantly shorter median survival compared to the ones in the most favorable molecular clusters $(0.70$ versus 2.05 years; (8.04 versus 24.6 months) $p=0.0024) .{ }^{86}$ Interestingly, when the different signatures previously identified by Phillips et al. were segregated into specific molecular clusters used by Gravendeel et al., one of the best prognostic clusters of Gravendeel is classified as poor prognostic group by Phillips. ${ }^{86}$ This discrepancy supports that while molecular profiling might be a promising alternative to histologic profiling, refinement is needed.

By integrating data from The Cancer Genome Atlas (TCGA), Verhaak et al. were able to subdivide GBM into four distinct subtypes based on patterns of gene expression, namely proneural, neural, classical, and mesenchymal. Each group differs in terms of genomic signature, age of the patient population, overall survival, and response to therapy (Table 3). ${ }^{36}$

Examination of responses to aggressive treatment per subtype showed that there is a significant reduction in mortality in the mesenchymal and classical subgroups. Aggressive treatment was defined in the study as concurrent chemoradiotherapy or more than three cycles of chemotherapy. Although a noted trend toward longer survival was found in the proneural subgroup compared to other types of GBM, it was found not to improve with aggressive treatments. ${ }^{36}$ In the analysis of the Rembrandt dataset by Guan et al., ${ }^{87}$ the proneural subtype was noted to be more prevalent in lower grade gliomas compared to GBMs. Though further research would be beneficial, these studies indicate a potential use for integrating methylation studies into glioma classification. 


\section{Table 2: Distinguishing genetic alterations and corresponding median survival in patients with various GBM molecular subtypes}

\begin{tabular}{l|l|l|l}
\hline $\begin{array}{l}\text { Molecular } \\
\text { subtype }\end{array}$ & Proneural & Proliferative & Mesenchymal \\
\hline $\begin{array}{l}\text { Distinguishing } \\
\text { genetic } \\
\text { alterations }\end{array}$ & $\begin{array}{c}\text { Intact PTEN, } \\
\text { normal EGFR; } \\
\text { no chromosome } \\
\text { gain or loss }\end{array}$ & $\begin{array}{l}\text { Gain of PIK3R3 } \\
\text { locus on } \\
\text { chromosome 1 }\end{array}$ & $\begin{array}{l}\text { Chromosome 7 gain, } \\
\text { chromosome } \\
10 \text { loss, PTEN } \\
\text { loss, EGFR } \\
\text { amplification }\end{array}$ \\
\hline Median survival & 174.5 weeks & 60.5 weeks & 65 weeks \\
\hline
\end{tabular}

Table 3: Frequency of common mutations in four molecular subtypes as identified by Verhaak et al. ${ }^{42}$ with frequencies

\begin{tabular}{l|c|c|c|c}
\hline $\begin{array}{l}\text { Molecular } \\
\text { subtype }\end{array}$ & $\begin{array}{c}\text { Proneural } \\
(\boldsymbol{\%})\end{array}$ & $\begin{array}{c}\text { Neural } \\
(\boldsymbol{\%})\end{array}$ & $\begin{array}{c}\text { Classical } \\
(\boldsymbol{\%})\end{array}$ & $\begin{array}{c}\text { Mesenchymal } \\
(\boldsymbol{\%})\end{array}$ \\
\hline TP53 & 54 & 21 & 0 & 32 \\
\hline NF1 & 5 & 16 & 5 & 37 \\
\hline IDH1 & $30^{*}$ & 5 & 0 & 0 \\
\hline PDGFRA & 11 & 0 & 0 & 0 \\
\hline EGFR & 16 & 26 & 32 & 5 \\
\hline
\end{tabular}

$* p$-Values at 0.01 level.

\section{Future Directions}

The advent of molecular testing has led to increased knowledge of glioma subgroups, each with its characteristic molecular associations and clinical features. More recently, multiplatform and machine-learning strategies to classify gliomas have showed promising results. For example, through multiplatform comprehensive analysis (DNA methylation, mRNA expression, microRNA expression, and long noncoding RNA expression), Mao et al. were able to identify differential expression in IDH-mutated versus wildtype astrocytic tumors as well as four signatures in the calcium signalling pathway of prognostic significance. ${ }^{88}$ Similar comprehensive analyses, though extensive, could allow for improved tumor classification beyond the 2016 WHO criteria. ${ }^{88}$ As molecular testing in gliomas is performed more routinely globally and as more is understood about the clinical significance of these tests, opportunities for targeted therapies will increase. In the era of personalized cancer treatments, knowledge of unique molecular characteristics which are specific to various types of gliomas can provide molecular targets for future drug development and refinement of current agents available.

In the future, the ability to reliably predict and prognosticate clinical responses and survival in patients with varying grades and classes of gliomas based on detailed molecular testing would be beneficial for clinical practice. It will not only allow for more informed decision-making for patients with this terminal disease, but also allow for health care professionals to tailor personalized treatment strategies to optimize patient outcomes.

\section{Conclusion}

The emergence of in-depth molecular profiling has significantly changed the field of neuro-oncology in recent years. Since the release of the 2016 WHO classification of CNS tumors, further information on classification, clinical outcomes, and potential changes to management of diffuse gliomas has been achieved. This paper aimed to summarize key knowledge of important glioma biomarkers to date, updates provided by the cIMPACT-NOW consortium, and important studies on DNA methylation testing in diffuse gliomas. As further studies on comprehensive molecular testing and its use in glioma classification are available, the landscape of glioma classification will continue to evolve to reflect clinically relevant tumor classes.

\section{CONFLICT OF INTEREST}

There are no disclosures for any of the above authors.

\section{Statement OF AUTHORShIP}

All persons meeting authorship criteria have been included above, and all authors certify that they have participated sufficiently. Each other certifies that the material or similar material has not been submitted or published in any other publications and has only been submitted to the Canadian Journal of Neurological Sciences.

Conception or design of the work: WM.

Data collection, analysis, and interpretation: NK, RO, SB.

Drafting the article: NK, RO, SB.

Critical revision of the article: WM.

Final approval of the version to be published: NK, RO, SB, WM.

\section{REFERENCES}

1. Ostrom QT, Gittleman H, Truitt G, Boscia A, Kruchko C, Barnholtz-Sloan JS. CBTRUS statistical report: primary brain and other central nervous system tumors diagnosed in the united states in 2011-2015. Neuro Oncol. 2018;20(Suppl. 4):iv1-86.

2. Stupp R, Mason WP, van den Bent, MJ, et al. Radiotherapy plus concomitant and adjuvant temozolomide for glioblastoma. N Engl J Med. 2005;352(10):987-96.

3. Louis DN, Ohgaki H, Wiestler OD, et al. The 2007 WHO classification of tumours of the central nervous system. Acta Neuropathol. 2007;114(2):97-109.

4. Louis DN, Perry A, Reifenberger G, et al. The 2016 World Health Organization classification of tumors of the central nervous system: a summary. Acta Neuropathol. 2016;131(6):803-20.

5. Parsons DW, Jones S, Zhang $\mathrm{X}$, et al. An integrated genomic analysis of human glioblastoma multiforme. Science. 2008; 321(5897):1807-12.

6. Cohen AL, Holmen SL, Colman H. IDH1 and IDH2 mutations in gliomas. Curr Neurol Neurosci Rep. 2013;13(5):345.

7. Yan H, Parsons DW, Jin G, et al. IDH1 and IDH2 mutations in gliomas. N Engl J Med. 2009;360(8):765-73.

8. Appin CL, Brat DJ. Molecular genetics of gliomas. Cancer J. 2014; 20(1):66-72.

9. Coons SW, Johnson PC, Scheithauer BW, Yates AJ, Pearl DK. Improving diagnostic accuracy and interobserver concordance in the classification and grading of primary gliomas. Cancer. 1997; 79(7):1381-93.

10. Kim W, Liau LM. IDH mutations in human glioma. Neurosurg Clin N Am. 2012;23(3):471-80.

11. Ueki K, Nishikawa R, Nakazato Y, et al. Correlation of histology and molecular genetic analysis of $1 \mathrm{p}, 19 \mathrm{q}, 10 \mathrm{q}$, TP53, EGFR, 
CDK4, and CDKN2A in 91 astrocytic and oligodendroglial tumors. Clin Cancer Res. 2002;8(1):196-201.

12. Maintz D, Fiedler K, Koopmann J, et al. Molecular genetic evidence for subtypes of oligoastrocytomas. J Neuropathol Exp Neurol. 1997;56(10):1098-104.

13. Brat DJ, Verhaak RGW, Aldape KD, et al. Comprehensive, integrative genomic analysis of diffuse lower-grade gliomas. N Engl J Med. 2015;372(26):2481-98.

14. Reifenberger G, Collins V, Hartmann C, et al. Oligoastrocytoma, NOS. In: Louis D, Ohgaki H, Wiestler O, Cavenee WK, editors. WHO classification of tumours of the central nervous system. International Agency for Research on Cancer; 2016. pp. 75-7.

15. Jin J, Hu C, Yu M, et al. Prognostic value of isocitrate dehydrogenase mutations in myelodysplastic syndromes: a retrospective cohort study and meta-analysis. PLoS ONE. 2014;9(6):e100206.

16. Dang L, Yen K, Attar EC. IDH mutations in cancer and progress toward development of targeted therapeutics. Ann Oncol. 2016; 27(4):599-608.

17. Choi C, Ganji SK, DeBerardinis RJ, et al. 2-Hydroxyglutarate detection by magnetic resonance spectroscopy in IDH-mutated patients with gliomas. Nat Med. 2012;18(4):624-9.

18. Gross S, Cairns RA, Minden MD, et al. Cancer-associated metabolite 2-hydroxyglutarate accumulates in acute myelogenous leukemia with isocitrate dehydrogenase 1 and 2 mutations. J Exp Med. 2010;207(2):339-44.

19. Yamashita AS, da Costa Rosa M, Borodovsky A, Festuccia WT, Chan T, Riggins GJ. Demethylation and epigenetic modification with 5-azacytidine reduces IDH1 mutant glioma growth in combination with temozolomide. Neuro Oncol. 2019;21(2): 189-200.

20. Metellus P, Coulibaly B, Colin C, et al. Absence of IDH mutation identifies a novel radiologic and molecular subtype of WHO grade II gliomas with dismal prognosis. Acta Neuropathol. 2010; 120(6):719-29.

21. Watanabe T, Nobusawa S, Kleihues P, Ohgaki H. IDH1 mutations are early events in the development of astrocytomas and oligodendrogliomas. Am J Pathol. 2009;174(4):1149-53.

22. Yan H, Bigner DD, Velculescu V, Parsons DW. Mutant metabolic enzymes are at the origin of gliomas. Cancer Res. 2009;69(24): 9157-9.

23. Wang $\mathrm{H}$, Tang $\mathrm{K}$, Liang $\mathrm{T}$, et al. The comparison of clinical and biological characteristics between IDH1 and IDH2 mutations in gliomas. J Exp Clin Cancer Res. 2016;35:86.

24. Hartmann C, Meyer J, Balss J, et al. Type and frequency of IDH1 and IDH2 mutations are related to astrocytic and oligodendroglial differentiation and age: a study of 1,010 diffuse gliomas. Acta Neuropathol. 2009;118(4):469-74.

25. Aldape K, Nejad R, Louis DN, Zadeh G. Integrating molecular markers into the World Health Organization classification of CNS tumors: a survey of the neuro-oncology community. Neuro Oncol. 2017;19(3):336-44.

26. Rizzo D, Ruggiero A, Martini M, Rizzo V, Maurizi P, Riccardi R. Molecular biology in pediatric high-grade glioma: impact on prognosis and treatment. Biomed Res Int. 2015;2015:215135.

27. Pollack IF, Hamilton RL, Sobol RW, et al. IDH1 mutations are common in malignant gliomas arising in adolescents: a report from the Children's Oncology Group. Childs Nerv Syst. 2011; 27(1):87-94.

28. Li S, Chou AP, Chen W, et al. Overexpression of isocitrate dehydrogenase mutant proteins renders glioma cells more sensitive to radiation. Neuro Oncol. 2013;15(1):57-68.

29. SongTao Q, Lei Y, Si G, et al. IDH mutations predict longer survival and response to temozolomide in secondary glioblastoma. Cancer Sci. 2012;103(2):269-73.

30. Houillier C, Wang X, Kaloshi G, et al. IDH1 or IDH2 mutations predict longer survival and response to temozolomide in lowgrade gliomas. Neurology. 2010;75(17):1560-6.

31. Sanson M, Marie Y, Paris S, et al. Isocitrate dehydrogenase 1 codon 132 mutation is an important prognostic biomarker in gliomas. J Clin Oncol. 2009;27(25):4150-54.

32. Wick W, Hartmann C, Engel C, et al. NOA-04 randomized phase III trial of sequential radiochemotherapy of anaplastic glioma with procarbazine, lomustine, and vincristine or temozolomide. J Clin Oncol. 2009;27(35):5874-80.

33. Hartmann C, Hentschel B, Tatagiba M, et al. Molecular markers in low-grade gliomas: predictive or prognostic? Clin Cancer Res. 2011;17(13):4588-99.

34. Weller M, Felsberg J, Hartmann C, Berger H, et al. Molecular predictors of progression-free and overall survival in patients with newly diagnosed glioblastoma: a prospective translational study of the German Glioma Network. J Clin Oncol. 2009;27(34): 5743-50.

35. Aibaidula A, Chan AK, Shi Z, et al. Adult IDH wild-type lowergrade gliomas should be further stratified. Neuro Oncol. 2017; 19(10): 1327-37.

36. Verhaak RGW, Hoadley KA, Purdom E, et al. Integrated genomic analysis identifies clinically relevant subtypes of glioblastoma characterized by abnormalities in PDGFRA, IDH1, EGFR, and NF1. Cancer Cell. 2010;17(1):98-110.

37. Ohgaki H, Kleihues P, Plate KH, Nakazato Y, Bigner DD. Giant cell glioblastoma. In: Louis D, Ohgaki H, Wiestler O, Cavenee WK, editors. WHO classification of tumours of the central nervous system. Lyon: International Agency for Research on Cancer; 2016, pp. 46-7.

38. Ellison DW, Kleinschmidt-DeMasters BK, Park SH. Epitheliod glioblastoma. In: Louis D, Ohgaki H, Wiestler O, Cavenee WK, editors. WHO classification of tumours of the central nervous system. Lyon: International Agency for Research on Cancer; 2016, pp. 50-1.

39. Burger PC, Giangaspero F, Ohgaki H, Biernat W. Gliosarcoma. In: Louis D, Ohgaki H, Wiestler O, Cavenee WK, editors. WHO classification of tumours of the central nervous system. Lyon: International Agency for Research on Cancerp; 2016, pp. 48-9.

40. Masui K, Mischel PS, Reifenberger G. Molecular classification of gliomas. Handb Clin Neurol. 2016;134:97-120.

41. Ballester LY, Huse JT, Tang G, Fuller GN. Molecular classification of adult diffuse gliomas: conflicting IDH1/IDH2, ATRX, and 1p/ 19q results. Hum Pathol. 2017;69:15-22.

42. Taal W, van der Rijt C CD, Dinjens WNM, et al. Treatment of large low-grade oligodendroglial tumors with upfront procarbazine, lomustine, and vincristine chemotherapy with long follow-up: a retrospective cohort study with growth kinetics. J Neurooncol. 2015;121(2):365-72.

43. van den Bent MJ Practice changing mature results of RTOG study 9802: another positive PCV trial makes adjuvant chemotherapy part of standard of care in low-grade glioma. Neuro Oncol. 2014;16(12):1570-4.

44. Vogazianou AP, Chan R, Bäcklund LM, et al. Distinct patterns of $1 \mathrm{p}$ and $19 q$ alterations identify subtypes of human gliomas that have different prognoses. Neuro Oncol. 2010;12(7):664-78.

45. Wiestler B, Capper D, Holland-Letz T, et al. ATRX loss refines the classification of anaplastic gliomas and identifies a subgroup of IDH mutant astrocytic tumors with better prognosis. Acta Neuropathol. 2013;126(3):443-51.

46. Gillet E, Alentorn A, Doukouré B, et al. TP53 and p53 statuses and their clinical impact in diffuse low grade gliomas. J Neurooncol. 2014;118(1):131-9.

47. Louis DN, Giannini C, Capper D, et al. cIMPACT-NOW update 2: diagnostic clarifications for diffuse midline glioma, H3 K27Mmutant and diffuse astrocytoma/anaplastic astrocytoma, IDH-mutant. Acta Neuropathol. 2018;135(4):639-42.

48. Nonoguchi N, Ohta T, Oh J, Kim Y, Kleihues P, Ohgaki H. TERT promoter mutations in primary and secondary glioblastomas. Acta Neuropathol. 2013;126(6):931-7.

49. Eckel-Passow JE, Lachance DH, Molinaro AM, et al. Glioma groups based on $1 \mathrm{p} / 19 \mathrm{q}$, IDH, and TERT promoter mutations in tumors. N Engl J Med. 2015;372(26):2499-508.

50. Simon M, Hosen I, Gousias K, et al. TERT promoter mutations: a novel independent prognostic factor in primary glioblastomas. Neuro Oncol. 2015;17(1):45-52.

51. Reuss DE, Kratz A, Sahm F, et al. Adult IDH wild type astrocytomas biologically and clinically resolve into other tumor entities. Acta Neuropathol. 2015;130(3):407-17. 
52. Chan AK, Yao Y, Zhang Z, et al. TERT promoter mutations contribute to subset prognostication of lower-grade gliomas. Mod Pathol. 2015;28(2):177-86.

53. Chan AK, Yao Y, Zhang Z, et al. Combination genetic signature stratifies lower-grade gliomas better than histological grade. Oncotarget. 2015;6(25):20885-901.

54. Vuong HG, Tran TTK, Ngo HTT, et al. Prognostic significance of genetic biomarkers in isocitrate dehydrogenase-wild-type lower-grade glioma: the need to further stratify this tumor entity - a meta-analysis. Eur J Neurol. 2019;26(3):379-87.

55. Brat DJ, Aldape K, Colman H, et al. cIMPACT-NOW update 3: recommended diagnostic criteria for "Diffuse astrocytic glioma, IDH-wildtype, with molecular features of glioblastoma, WHO grade IV". Acta Neuropathol. 2018;136(5):805-10.

56. Horbinski C. To BRAF or not to BRAF: is that even a question anymore? J Neuropathol Exp Neurol. 2013;72(1):2-7.

57. Cantwell-Dorris ER, O'Leary JJ, Sheils OM. BRAFV600E: implications for carcinogenesis and molecular therapy. Mol Cancer Ther. 2011;10(3):385-94.

58. Becker AP, Scapulatempo-Neto C, Carloni AC, et al. KIAA1549: BRAF gene fusion and FGFR1 hotspot mutations are prognostic factors in pilocytic astrocytomas. J Neuropathol Exp Neurol. 2015;74(7):743-54.

59. Jeuken JWM, Wesseling P. MAPK pathway activation through BRAF gene fusion in pilocytic astrocytomas; a novel oncogenic fusion gene with diagnostic, prognostic, and therapeutic potential. J Pathol. 2010;222(4):324-8.

60. Collins VP, Jones DTW, Giannini C. Pilocytic astrocytoma: pathology, molecular mechanisms and markers. Acta Neuropathol. 2015; 129(6):775-88.

61. Schindler G, Capper D, Meyer J, et al. Analysis of BRAF V600E mutation in 1320 nervous system tumors reveals high mutation frequencies in pleomorphic xanthoastrocytoma, ganglioglioma and extra-cerebellar pilocytic astrocytoma. Acta Neuropathol. 2011;121(3):397-405.

62. Kleinschmidt-DeMasters BK, Aisner DL, Birks DK, Foreman NK. Epithelioid GBMs show a high percentage of BRAF V600E mutation. Am J Surg Pathol. 2013;37(5):685-98.

63. Dahiya S, Emnett RJ, Haydon DH, et al. BRAF-V600E mutation in pediatric and adult glioblastoma. Neuro Oncol. 2014;16(2): 318-9.

64. Mackay A, Burford A, Molinari V, et al. Molecular, pathological, radiological, and immune profiling of non-brainstem pediatric high-grade glioma from the HERBY phase II randomized trial. Cancer Cell. 2018;33(5):82-842.e5.

65. Kleinschmidt-DeMasters BK, Mulcahy Levy JM. H3 K27M-mutant gliomas in adults vs. children share similar histological features and adverse prognosis. Clin Neuropathol. 2018;37(2):53-63.

66. Solomon DA, Wood MD, Tihan T, et al. Diffuse midline gliomas with histone $\mathrm{H} 3-\mathrm{K} 27 \mathrm{M}$ mutation: a series of 47 cases assessing the spectrum of morphologic variation and associated genetic alterations. Brain Pathol. 2016;26(5):569-80.

67. Castel D, Philippe C, Kergrohen T, et al. Transcriptomic and epigenetic profiling of 'diffuse midline gliomas, H3 K27Mmutant' discriminate two subgroups based on the type of histone H3 mutated and not supratentorial or infratentorial location. Acta Neuropathol Commun. 2018;6(1):117.

68. Karremann M, Gielen GH, Hoffmann M, et al. Diffuse high-grade gliomas with H3 K27M mutations carry a dismal prognosis independent of tumor location. Neuro-oncology. 2018;20(1): 123-31.

69. López G, Oberheim Bush NA, Berger MS, Perry A, Solomon DA. Diffuse non-midline glioma with H3F3A K27M mutation: a prognostic and treatment dilemma. Acta Neuropathol Commun. 2017;5(1):38.

70. Castel D, Philippe C, Calmon R, et al. Histone H3F3A and HIST1H3B K27M mutations define two subgroups of diffuse intrinsic pontine gliomas with different prognosis and phenotypes. Acta Neuropathol. 2015;130(6):815-27.

71. von Deimling A, Korshunov A, Hartmann C. The next generation of glioma biomarkers: MGMT methylation, BRAF fusions and IDH1 mutations. Brain Pathol. 2011;21(1):74-87.

72. Hegi ME, Diserens A, Gorlia T, et al. MGMT gene silencing and benefit from temozolomide in glioblastoma. $\mathrm{N}$ Engl J Med. 2005;352(10):997-1003.

73. Esteller M, Garcia-Foncillas J, Andion E, et al. Inactivation of the DNA-repair gene MGMT and the clinical response of gliomas to alkylating agents. N Engl J Med. 2000;343(19):1350-54.

74. Nakamura M, Watanabe T, Yonekawa Y, Kleihues P, Ohgaki H. Promoter methylation of the DNA repair gene MGMT in astrocytomas is frequently associated with $\mathrm{G}: \mathrm{C} \rightarrow \mathrm{A}: \mathrm{T}$ mutations of the TP53 tumor suppressor gene. Carcinogenesis. 2001;22(10): 1715-19.

75. Paul Y, Mondal B, Patil V, Somasundaram K. DNA methylation signatures for 2016 WHO classification subtypes of diffuse gliomas. Clin Epigenetics. 2017;9:32.

76. Weller M, Pfister SM, Wick W, Hegi ME, Reifenberger G, Stupp R. Molecular neuro-oncology in clinical practice: a new horizon. Lancet Oncol. 2013;14(9):370.

77. Ordway JM, Bedell JA, Citek RW, et al. Comprehensive DNA methylation profiling in a human cancer genome identifies novel epigenetic targets. Carcinogenesis. 2006;27(12):2409-23.

78. Noushmehr H, Weisenberger DJ, Diefes K, et al. Identification of a $\mathrm{CpG}$ island methylator phenotype that defines a distinct subgroup of glioma. Cancer Cell. 2010;17(5):510-22.

79. Jones PA, Baylin SB. The epigenomics of cancer. Cell. 2007; 128(4):683-92.

80. Toyota M, Ahuja N, Ohe-Toyota M, Herman JG, Baylin SB, Issa JP. $\mathrm{CpG}$ island methylator phenotype in colorectal cancer. Proc Natl Acad Sci U S A. 1999;96(15):8681-6.

81. van den Bent MJ, Gravendeel LA, et al. A hypermethylated phenotype is a better predictor of survival than MGMT methylation in anaplastic oligodendroglial brain tumors: a report from EORTC study 26951. Clin Cancer Res. 2011;17(22):7148-55.

82. Ceccarelli M, Barthel FP, Malta TM, et al. Molecular profiling reveals biologically discrete subsets and pathways of progression in diffuse glioma. Cell. 2016;164(3):550-63.

83. Kloosterhof NK, de Rooi JJ, Kros M, et al. Molecular subtypes of glioma identified by genome-wide methylation profiling. Genes Chromosomes Cancer. 2013;52(7):665-74.

84. de Souza CF, Sabedot TS, Malta TM, et al. A distinct DNA methylation shift in a subset of glioma $\mathrm{CpG}$ Island methylator phenotypes during tumor recurrence. Cell Rep. 2018;23(2): 637-51.

85. Phillips HS, Kharbanda S, Chen R, et al. Molecular subclasses of high-grade glioma predict prognosis, delineate a pattern of disease progression, and resemble stages in neurogenesis. Cancer Cell. 2006;9(3):157-73.

86. Gravendeel LAM, Kouwenhoven MCM, Gevaert O, et al. Intrinsic gene expression profiles of gliomas are a better predictor of survival than histology. Cancer Res. 2009;69(23):9065-72.

87. Guan X, Vengoechea J, Zhang S, et al. Molecular subtypes of glioblastoma are relevant to lower grade glioma. PLoS One. 2014;9(3):e91216.

88. Mao C, Yin J, Zhang Y, et al. The molecular classification of astrocytic tumors. Oncotarget. 2017;8(56):96340-350. 